\title{
As principais consequências dos resíduos sólidos sobre o meio ambiente e a saúde da população no município de cabaret-haiti
}

The main consequences of solid waste on the environment and the health of the population in the municipality of CabaretHaiti

Las principales consecuencias de los residuos sólidos sobre el medio ambiente y la salud de la población en el municipio de Cabaret-Haití

\author{
Ralph Charles ${ }^{1}$ \\ Regina Célia de Oliveira ${ }^{2}$ \\ Pedro Spanghero ${ }^{3}$ \\ Universidade Estadual de Campinas \\ São Paulo, Brasil
}

1 Mestrando em Geografia, Universidade Estadual de Campinas, São Paulo, Brasil. Correo electrónico: cralph001@yahoo.fr

2 Professora, doutoura, Universidade Estadual de Campinas, São Paulo, Brasil. Correo electrónico: reginacoliveira@unicamp.br

3 Mestrando em Geografia, Universidade Estadual de Campinas, São Paulo, Brasil. Correo electrónico: Geo.pedro@gmail.com 
Ralph Charles, Regina Célia de Oliveira, Pedro Spanghero. The main consequences of solid waste on the environment and the health of the population in the municipality of Cabaret-Haiti

\title{
Resumo
}

A gestão de resíduos sólidos para um mundo mais sustentável está presente sempre nas conferencias mundiais sobre o meio ambiente, sendo que, é uma estratégia para conciliar um desenvolvimento com proteção dos ecossistemas, considerando que a geração e disposição final dos resíduos sólidos urbanos é hoje um dos maiores problemas da sociedade moderna. Um dos reflexos dessa negligência é percebido facilmente entre a população, como no caso da carência do saneamento básico, o que prejudica além do próprio meio ambiente, a saúde humana com a contaminação do solo, dos corpos d'água, os alimentos e a qualidade do ar, implicando em inúmeros casos de doenças. Esses resíduos podem ser fontes financeiras, sendo que, podem ser reutilizados para outros fins, ao mesmo tempo, podem ser considerados como perigo para a sustentabilidade do planeta. Dessa forma, este artigo tem por objetivo apresentar cenários de eventos que tem provocado alterações ambientais na dinâmica de uso da terra no município de Cabaret-Haiti, sobretudo através da percepção da população sobre a gestão de resíduos sólidos e a deposição inadequada do lixo, visando preservar e recuperar a qualidade ambiental propicia à vida.

Palavras-chave: Resíduos Sólidos; Mudanças Climáticas; Sustentabilidade do Planeta; Reciclagem; Recursos Naturais.

\begin{abstract}
Solid waste management for a more sustainable world is always present at world conferences on the environment, and is a strategy to reconcile development with protection of ecosystems, considering that the generation and final disposal of urban solid waste is now a days a of the major problems of modern society. One of the reflexes of this neglect is easily perceived among the population, as in the case of the lack of basic sanitation, which damages beyond the environment itself, human health with contamination of soil, bodies of water, food and quality of the air, implying in numerous cases of diseases. These residues can be financial sources, and can be reused for other purposes, at the same time, can be considered as a danger to the sustainability of the planet. Thus, this article aims to present scenarios of events that have caused environmental changes in the dynamics of land use in Cabaret-Haiti, mainly through the perception of the population on the management of solid waste and the improper disposal of garbage, aiming at preserving and restoring the environmental quality conducive to life.
\end{abstract}

Keyword: Solid Waste; Climate changes; Sustainability of the Planet; Recycling; Natural resources.

\section{Resumen}

La gestión de residuos sólidos para un mundo más sostenible siempre está presente en las conferencias mundiales sobre el medio ambiente, y es una estrategia para conciliar el desarrollo con la protección de los ecosistemas, considerando que la generación y disposición final de los desechos sólidos urbanos es uno de los principales problemas. de la sociedad moderna. Uno de los reflejos de este abandono se percibe fácilmente entre la población, como en el caso de la falta de saneamiento básico, que daña más allá del propio medio ambiente, la salud humana con contaminación del suelo, cuerpos de agua, comida y calidad del aire, lo que implica en numerosos casos de enfermedades. Estos residuos pueden ser fuentes financieras, y pueden reutilizarse para otros fines, al mismo tiempo, pueden considerarse como un peligro para la sostenibilidad del planeta. Por lo tanto, este artículo tiene como objetivo presentar escenarios de eventos que han causado cambios ambientales en la dinámica del uso de la tierra en Cabaret-Haití, principalmente a través de la percepción de la población sobre el manejo de residuos sólidos y la eliminación inadecuada de basura, con el objetivo de preservar y restaurar la calidad ambiental propicia para la vida.

Palabras clave: Residuos sólidos; Cambios climáticos; Sostenibilidad del Planeta; reciclaje; Recursos naturales. 
Ralph Charles, Regina Célia de Oliveira, Pedro Spanghero. As principais consequências dos resíduos sólidos sobre o meio ambiente e a saúde da população no município de cabaret-haiti

\section{Introducão}

O crescimento no número e na intensidade dos impactos causados pela alteração da paisagem que desenvolvem os chamados desastres naturais releva uma importância muito grande para estudos socioeconômicos. Com a globalização da informação, todos os dias destacam-se notícias de um evento com perda de vidas e matérias motivadas pela exposição de populações e elementos da natureza (ONU, 2010).

A demografia e as mudanças socioeconômicas que caracterizam os últimos 30 anos nos países indústrias indicam um aumento nas populações urbanas, que consequentemente atinge um número maior de pessoas e bens materiais no sentido de exposição a desastres naturais como, tsunamis, escorregamentos de terras, terremotos e inundações. Esse cenário de inúmeros riscos presentes no espaço geográfico é o objeto de estudo de trabalhos sobre vulnerabilidade. Sobretudo em países subdesenvolvidos que detém de clima tropical ou equatorial submetido alto índices pluviométricos, como em estudos de caso na América Latina, África, Caribe e sudeste asiático, a caracterização de bacias hidrográficas tem despontado como levantamento de informações (diagnósticos) e leitura de ambientes voltados para compreender como os fenômenos naturais geram (ou irá gerar) consequências negativas imediatas nas perdas materiais e vidas humanas (AMORIM e PRADES, 2017).

As decisões que envolvem o gerenciamento de resíduos sólidos urbanos são fundamentalmente decisões sobre saúde pública e requerem, portanto, a integração entre políticas econômicas, sociais e ambientais. O complexo desafio para as grandes cidades na gestão de resíduos sólidos neste início de século pode ser enfrentado pela formulação de políticas públicas que objetivem eliminar os riscos à saúde e ao ambiente, que colaborem na mitigação das mudanças climáticas relacionadas à ação humana e, ao mesmo tempo, garantam a inclusão social efetiva de parcelas significativas da população (AMORIM e OLIVEIRA, 2007).

Pensando na melhor forma de lidar com os problemas ambientais, a questão dos resíduos vem sendo apontada pelos ambientalistas como um dos mais graves problemas ambientais urbanos da atualidade. A compreensão da necessidade do gerenciamento integrado dos resíduos sólidos propiciou a criação da chamada Política dos $3 R^{\text {ee }}$ (Reduzir, Reutilizar e Reciclar), que inspira técnica e pedagogicamente os meios de enfrentamento 
Ralph Charles, Regina Célia de Oliveira, Pedro Spanghero. The main consequences of solid waste on the environment and the health of the population in the municipality of Cabaret-Haiti

da questão do lixo conforme afirma o Instituto Haitiano de Estatística e de Informática (IHSI), 2010.

Partimos do pressuposto de que a deposição de forma inadequada desses resíduos podem ser os resultados dos problemas ambientais, e tem consequências negativas na saúde populacional, este trabalho tem como objetivo fazer uma breve apresentação do estado ambiental e o impacto dos resíduos sólidos sobre a saúde da população no município de Cabaret.

\section{Metodologia}

O trabalho adotou a metodologia apontada por Rodrigues, silva \& Calvacanti (2010). O procedimento metodológico consiste em primeiro lugar, na realização de um trabalho de campo em Cabaret para reviver essa situação. A área de estudo é o centro do município, sendo que, é uma área urbana que concentra grandes atividades econômicas como o mercado municipal. Durante a atividade no campo, foram entrevistados duzentas (200) pessoas, as perguntas foram direcionadas para o entendimento da gestão diária do lixo, para obter dados primários, ou seja, a impressão da população sobre os problemas ambientais na cidade.

Dentro os entrevistados, 117 pessoas eram de sexo feminino $(59 \%)$ e 83 são masculinos (41\%). A escolha foi feita de forma aleatória, sendo direcionado para quem estava na casa no momento da entrevista. As perguntas foram direcionadas para o entendimento da percepção ambiental da população sobre os problemas relacionados com o lixo.

Do ponto de vista cartográfico, foi elaborado um mapa de uso da terra da área de estudo, na escala 1:10.000, utilizando imagens orbitais com o uso, disponibilizada no Google Earth, manipulada em ambinete Arc Gis 10.1. Na elaboração do mapa de uso, foi utilizada a classificação não supervisionada de forma manual, delimitando as unidades da paisagem que apresentem sistemas semelhantes de funcionamento.

\section{Breve histórico da Área de Estudo}

De acordo com l'Institut Haitien de Statistique et d'Informatique IHSI 2010, (traduzido em português como: Instituto Haitiano de Estatístico e de informático) o município de Cabaret está localizado no departamento Oeste da República do Haiti. Situado a 35 kilômetros $(\mathrm{km})$ de Porto príncipe a capital haitiana, no arrondissement de Arcahaie. Geograficamente, 
Ralph Charles, Regina Célia de Oliveira, Pedro Spanghero. As principais consequências dos resíduos sólidos sobre o meio ambiente e a saúde da população no município de cabaret-haiti

Cabaret é delimitada a norte pelos municípios de Arcahaie e Saut-d'eau; a sul pelo Mar do Caribe; a leste pelos municípios do Croix-des-Bouquets e Saut-d'eau e a oeste pela cidade de Arcahaie e o Mar do Caribe.

Figura 1. Mapa de localização da área de estudos $\left(18^{\circ} 44^{\prime} 0^{\prime \prime} \mathrm{N} 72^{\circ} 25^{\prime} 0^{\prime \prime} \mathrm{W}\right)$
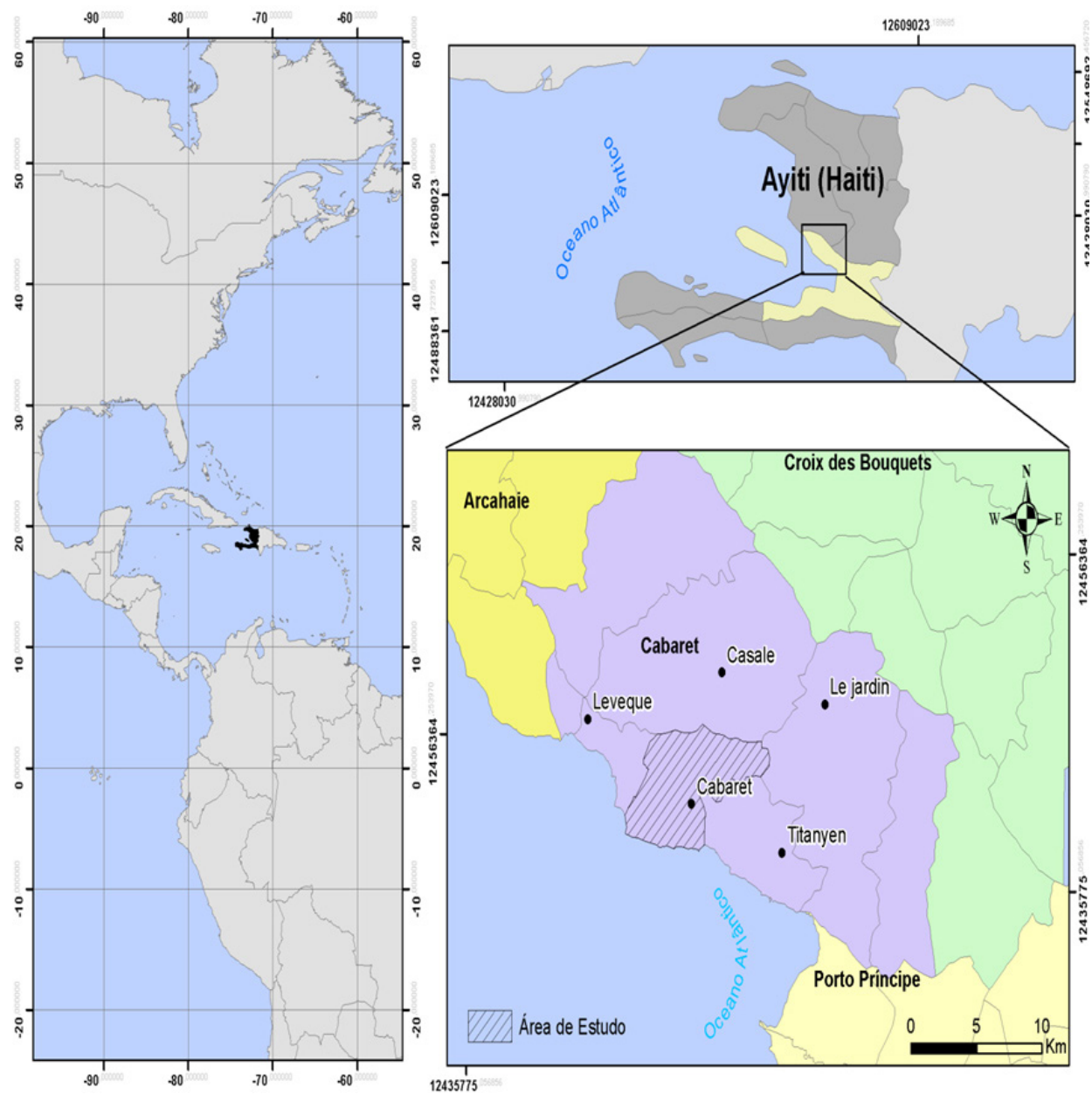

Fonte: Elaborado pelo autor, baseando nos dados de USGS 2017.

O município possui uma área de $204.89 \mathrm{~km} 2$ e está dividido em 4 seções comunais: 1ere section Boucassin, 2eme section Boucassin, 3eme section source Matelas, $4^{\text {eme }}$ section Fonds des Blancs. De acordo com o censo de 2010 do IHSI, o município de Cabaret tem uma população de 
70.000 habitantes, o que dá uma densidade de 346 habitantes por quilômetro quadrado, distribuídos tanto nas zonas rurais e urbanas. Quase 78\% dessa população vivem em áreas rurais. Cerca da metade $(51,7 \%)$ da população do município são mulheres. A escassez de homens é muito mais pronunciada nas áreas urbanas (86 homens para cada 100 mulheres) do que nas áreas rurais ( 95 homens para cada 100 mulheres). A distribuição da população é representada da forma seguinte: $68 \%$ são pessoas com idades entre 15-64 anos, e 32\% são pessoas em cima de 65 anos (IHSI, 2010).

O município foi construído pelo Estado haitiano a década de 60: era uma cidade moderna com arruamentos simétricos, uma referência para os padrões urbanos do estado. Um exemplo da qualidade da infraestrutura citadina era que, em períodos chuvosos, as áreas de planície não eram tomadas por enchentes. No entanto, a partir dos anos 80, a instabilidade política teve um impacto considerável no município de Cabaret, particularmente o centro velho passa a ser significativamente alterado por um crescimento urbano desordenado. Cabaret foi considerado como município em 21 de junho de 1933, sob o governo do presidente Stenio Vincent e, é a porta de entrada para "les cotes des Arcadins" um importante centro turístico. De forma administrativa e financeira, as principais atividades em Cabaret são agricultura e comercio. O município de Cabaret possui um clima tropical, exceto la $4^{\mathrm{eme}}$ section que apresenta um clima mais fresco devido a sua altitude, sendo que, é a parte mais elevada do município, e devido a sua localização, esta área recebe entre 800 e $1800 \mathrm{~mm}$ de precipitação média annual (IHSI, 2010).

\section{Vulnerabilidade socioambiental da população frente aos riscos e desastres}

O município de Cabaret apresenta historicamente um elevado grau de vulnerabilidade ambiental, conforme os dados da Organização das Nações Unidas (ONU) 2008, com destaque para os problemas ligados a falta de saneamento básico. Entretanto, o cenário de desastres consolidou-se em Setembro de 2008, quando Cabaret sofreu a passagem dos furacões Ike e Hanna, que causou danos significativos ao cenário urbano resultando em os seguintes números de perdas: 71 mortos, 20 desaparecidos, 410 casas destruídas, 530 casas danificadas, 6530 famílias desabrigadas, 1.250 hectares de terras plantadas com bananas, milho, mandioca, milho destruídos. 
Ralph Charles, Regina Célia de Oliveira, Pedro Spanghero. As principais consequências dos resíduos sólidos sobre o meio ambiente e a saúde da população no município de cabaret-haiti

As residências que não foram destruídas ou danificadas foram invadidas de lama, forçando os seus proprietários a uma limpeza imediata para continuar suas vidas se não quisessem ir aos abrigos provisórios. Assim de acordo com a Direção da Proteção Civil, o município está se tornando um lugar simbólico de desastres naturais no país (ONU, 2008).

Sendo assim, com o tempo e as condições meteorológicas desfavoráveis, Cabaret revela ainda mais a sua vulnerabilidade. Em outubro de 2007, essa região havia sofrido graves inundações. O último relatório da Direção de Proteção Civil de 2008 revelou mais de 20 pessoas perderam suas vidas fora das grandes perdas socioeconômicas e ambientais. Os danos já causados pela temporada dos furacões só confirma a fragilidade ambiental dessa área. Mas não é só o município de Cabaret que pode ser considerado como um símbolo do desastre, é o país todo, pois o Haiti conheceu durante pelo menos um mês quatros grandes tempestades tropicais: Hanna, Ike, Gustave e Fay. A organização das Nações Unidas (ONU, 2008) afirma que 800000 pessoas foram afetadas, o que representa $10 \%$ da população (ONU, 2008).

O município de Cabaret não foi poupado pelo terremoto de 2010 que devastou várias localidades e quebrou a infraestrutura local. Houve 72 mortos, 13 desaparecidos e 2.566 feridos. Mais de 3.500 casas foram destruídas e cerca de 10.000 danificados. Um número de 183 instituições públicas e privadas foram danificados ou destruídos nas quatro seções comunais. Apesar dos danos, o município conheceu um grande fluxo migratório sendo que Cabaret recebeu mais de 2000 pessoas da área metropolitana de Porto Príncipe duramente atingida pelo terremoto, em busca de melhor qualidade de vida (IHSI, 2010).

\section{Uso da terra urbano e a percepção da população sobre resíduos sólidos}

A gestão adequada de lixo é o maior desafio encontrado no município de Cabaret desde a década 80, sendo que, este lugar começou a receber grande fluxos de pessoas, devido a sua proximidade da capital do país que é o lugar onde é localizado todos os ministérios, os grande infraestruturas assim como os serviços. Isso faz com que o consumo de alimento aumentou de forma exponencial e Cabaret assim como o país todo não possui empresas que atuam na gestão e tratamento de lixo. Como resultado, vimos a deposição de resíduos sólidos urbanos de forma inadequada nas ruas, nos 
Ralph Charles, Regina Célia de Oliveira, Pedro Spanghero. The main consequences of solid waste on the environment and the health of the population in the municipality of Cabaret-Haiti

rios em qualquer lugar no município. A figura II traz um exemplo de lixo depositado a céu aberto no centro do município.

Figura 2. Depositado no centro do município de forma inadequada

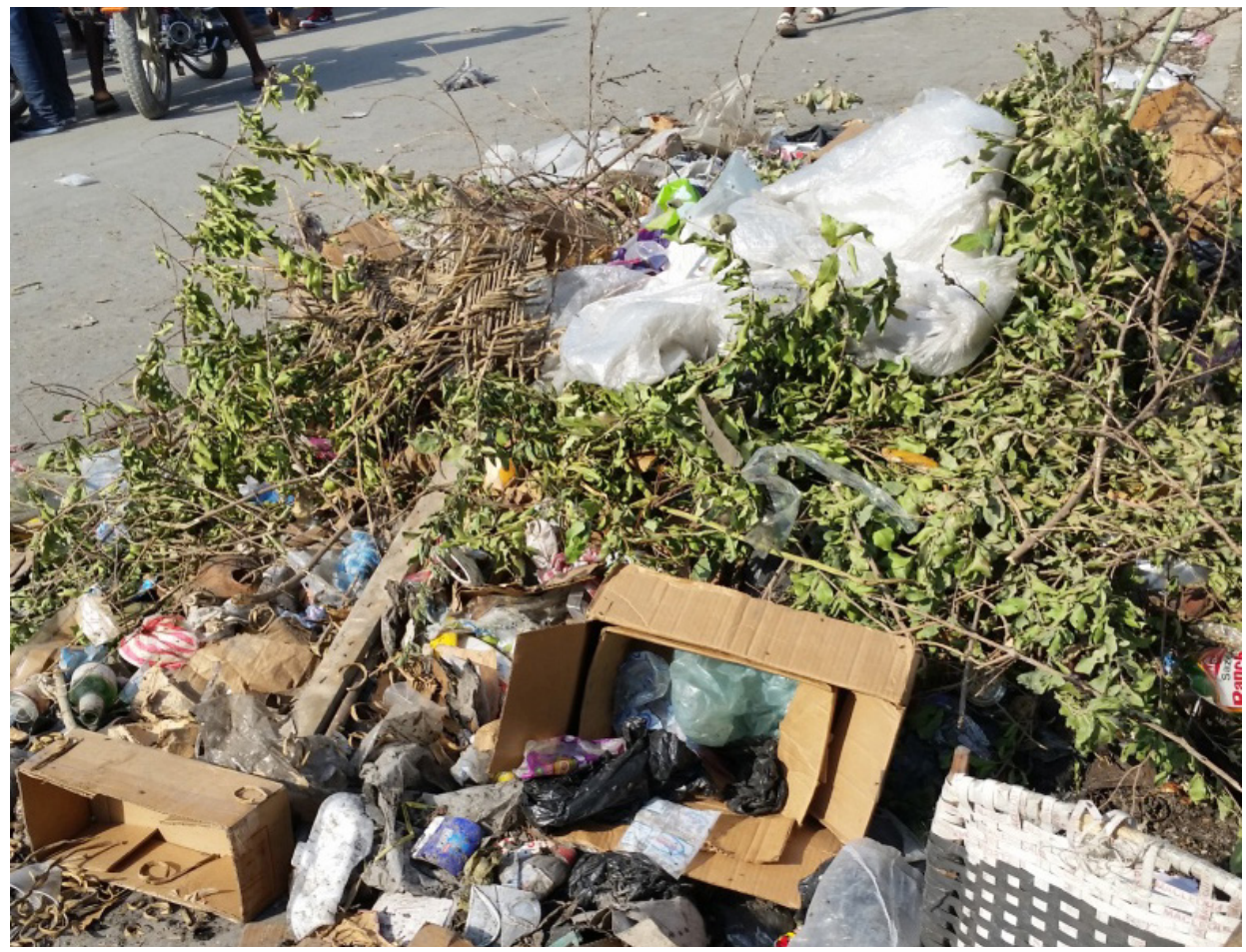

Foto: Ralph Charles, janeiro de 2017

O uso da terra em Cabaret é efeito de forma desordenado. O poder público não tem o controle desta situação, sendo que, na prática os donos de terras são quem controlam. O problema de uso inadequado da terra não é presente somente no município de Cabaret, mas nos dez (10) departamentos da república do Haiti. As zonas consideradas como zonas de riscos estão sendo ocupadas pela população que migra para Cabaret depois do terremoto de 2010. Isso dificulta ainda mais a questão de saneamento no município e até o campo de futebol que é uma área atrativa torna zona de deposição de lixo (PIMVP, 2001).

O mapa seguinte apresenta essa situação com mais detalhes. 
Ralph Charles, Regina Célia de Oliveira, Pedro Spanghero. As principais consequências dos resíduos sólidos sobre o meio ambiente e a saúde da população no município de cabaret-haiti

Figura 3. Mapa de uso da terra e deposição de lixo e massa na área urbana em Cabaret

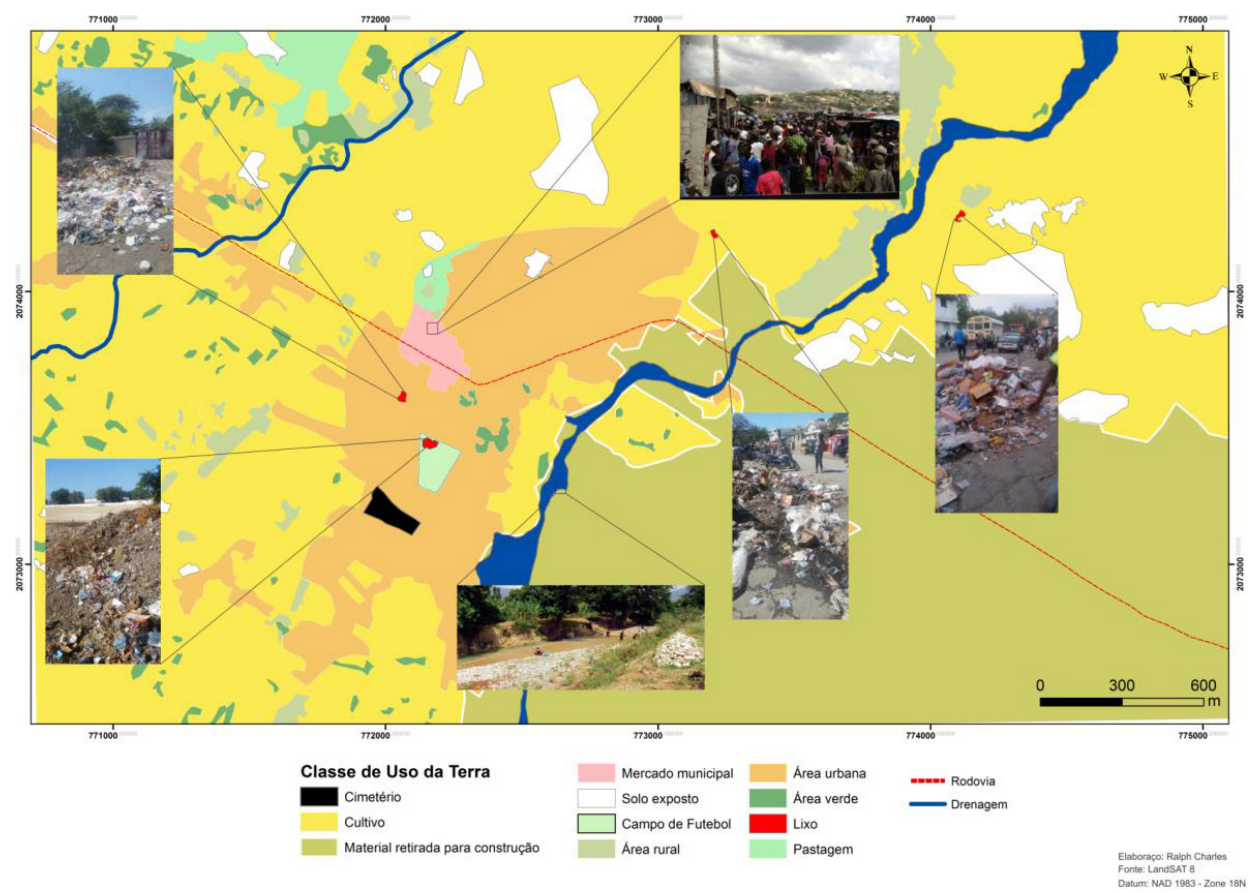

Fonte: Elaborado pelo autor

Como mostra a figura IV, Cabaret é uma zona costeira, os resíduos muitas vezes são jogados nos rios. Esses resíduos possuem grande capacidade de dispersão por ondas, correntes e ventos, podendo ser encontrados no meio dos oceanos e em áreas remotas. O problema, contudo, se torna mais aparente nas zonas onde as atividades humanas estão concentradas. Foram encontrados nessas zonas principalmente plásticos, poluentes orgânicos persistentes, metais pesados e nitrogênio, bem como ratificado. Parece que o compromisso de agir para reduzir a incidência e impacto destes poluentes no ecossistema marinho ainda é um grande desafio para o poder público. 
Ralph Charles, Regina Célia de Oliveira, Pedro Spanghero. The main consequences of solid waste on the environment and the health of the population in the municipality of Cabaret-Haiti

Figura 4. (18 $\left.43^{\prime} 40^{\prime \prime} \mathrm{N}, 072^{\circ} 27^{\prime} 05^{\prime \prime}\right)$ : Lixo nas zonas costeiras.

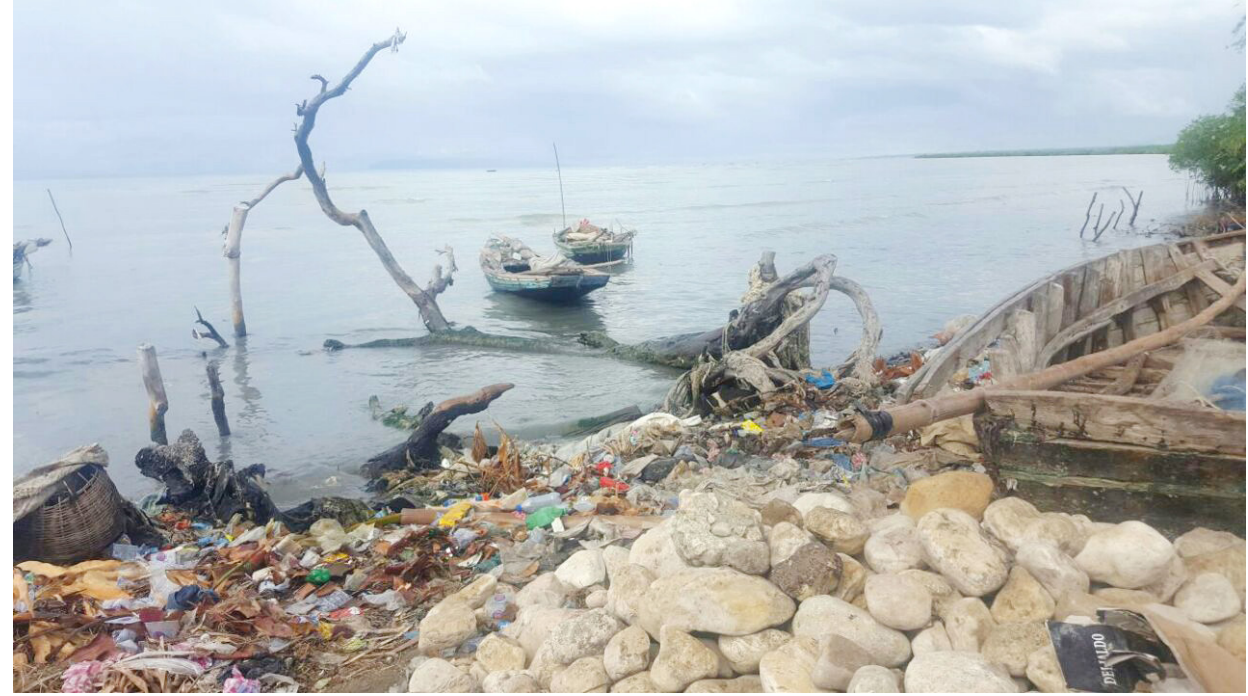

Foto: Ralph Charles, janeiro de 2017

Os problemas gerados pelo descarte inadequado dos resíduos sólidos são muitos e, geralmente, visíveis. Na maioria dos casos, eles se configuram como agressões ambientais e até como uma questão sanitária que coloca em risco à saúde pública. Os resíduos sólidos apresentam atualmente um dos maiores perigos que afetam a qualidade de vida em muitos países, ou seja, a vida de milhões de pessoas no mundo, desde a sua produção até o destino final. Um dos maiores desafios com que se defronta a sociedade moderna é o equacionamento da questão do lixo urbano.

Apesar da grande expansão da ciência e da tecnologia, hoje em dia é muito comum encontrar nas ruas, montanhas de lixo no Haiti, assim como em outros países de terceiro mundo. Isso pelo fato de que em vários países do mundo não há ainda o controle do lixo produzido diariamente, sendo assim, não há também como fazer a gestão destes resíduos sólidos de forma adequada. Segundo IBGE (2010), esses tipos de lixo representa problemas muito graves para o meio ambiente e também consequências negativas sobre a saúde da população (IBGE, 2010).

Infelizmente, O Ministério da Saúde Pública e da População (MSPP) identifica o lixo a céu aberto como fonte de alimentação para a sobrevivência da população mais pobre. Por outro lado, animais também utilizam o 
Ralph Charles, Regina Célia de Oliveira, Pedro Spanghero. As principais consequências dos resíduos sólidos sobre o meio ambiente e a saúde da população no município de cabaret-haiti

lixo como local de alimentação, reprodução e abrigo. Uma das consequências é a proliferação de diversas doenças, sendo que esses vermes servem como intermediários na propagação de algumas doenças e os transmitem para outros seres, como o homem (MSPP, 2007).

Na verdade, percebemos que o problema de gestão inadequado dos resíduos sólidos é a base de todos os problemas ambientais que o município de Cabaret está enfrentando desde a década de oitenta até os dias atuais. Os eventos extremos precitados estão relacionados entre si e são resultados do grau de fragilidade, assim com a vulnerabilidade socioambiental da cidade.

Devido à falta de infraestrutura básica na cidade, não existe uma forma adequada de lidar com o lixo. O poder público não mostra grandes interesses com a destinação final dos resíduos sólidos urbanos. Assim a população usa várias formas empíricas a fim de se livrar do lixo produzido diariamente. As principais formas são a queimada, outros jogam na rua, uma parte da população traz o lixo para o ponto de coleta apesar de que não existe na prática ponto de coleta definindo o acumulo do lixo na rua, e existe também outra forma muito comum que é jogar o lixo na rua.

De acordo com a população local, o lixo está na base de vários problemas de saúde. Durante a entrevista, com a pergunta, você acha que o lixo pode trazer consequenciais negativas sobre a saúde? O objetivo dessa questão foi verificar a percepção do entrevistado quanto a relação do lixo e saúde da população. Assim, 38 pessoas responderam que a febre é uma doença ligada aos lixos lançados nas ruas. Com a mesma pergunta, 35 acham que a gripe é resultado do mau tratamento do lixo. Por outro lado, 105 pessoas responderam que o problema respiratório que a maioria da população está enfrentando é por causa dos lixos que liberam gases na atmosfera e isso contamina o ar. Enfim, 28 responderam que o lixo pode trazer outras consequências na saúde populacional.

A figura $\mathrm{V}$ representa as respostas da população durante a entrevista sobre os principais problemas de saúde que o lixo pode trazer. 
Ralph Charles, Regina Célia de Oliveira, Pedro Spanghero. The main consequences of solid waste on the environment and the health of the population in the municipality of Cabaret-Haiti

Figura 5. Problemas de saúde relacionados ao lixo

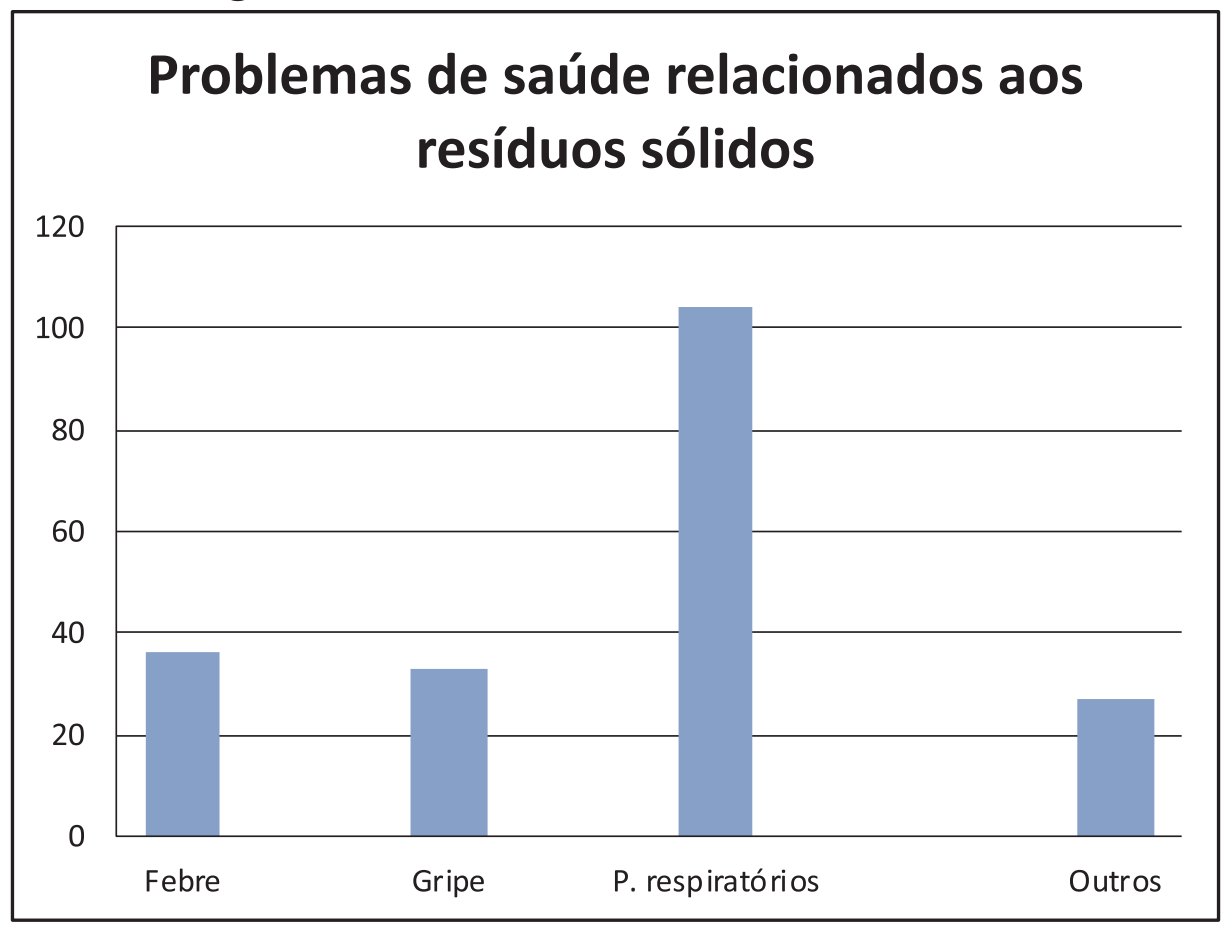

Fonte: Elaborado pelo autor através os dados do campo.

Para uma jovem entrevistada de 27 anos, em que a sua família mora em Cabaret há mais que 20 anos, ela percebe que o problema de lixo não é tão complicado se for comparado com a situação do lixo na capital do país onde a situação é caótica. Para ela Cabaret tem tudo para superar o problema de lixo, só que precisa de todo mundo para melhorar essa situação. Ela percebe uma falta de consciência e de amor, "porque nos não protegemos algo si não gostamos". Nesse sentido a gestão dos resíduos sólidos no município deve começar pela prefeitura até a menor da cidade, explica ela.

A figura VI representa as diferentes respostas com a pergunta por que tem tanto lixo nas ruas no município de cabaret? As respostas são dadas de acordo com o grau de escolaridade, mas é importante reparar que as repostas são muito interessantes seja das intelectuais e dos analfabetos. 
Ralph Charles, Regina Célia de Oliveira, Pedro Spanghero. As principais consequências dos resíduos sólidos sobre o meio ambiente e a saúde da população no município de cabaret-haiti

Figura 6. Opinião das pessoas entrevistadas sobre o lixo na rua.

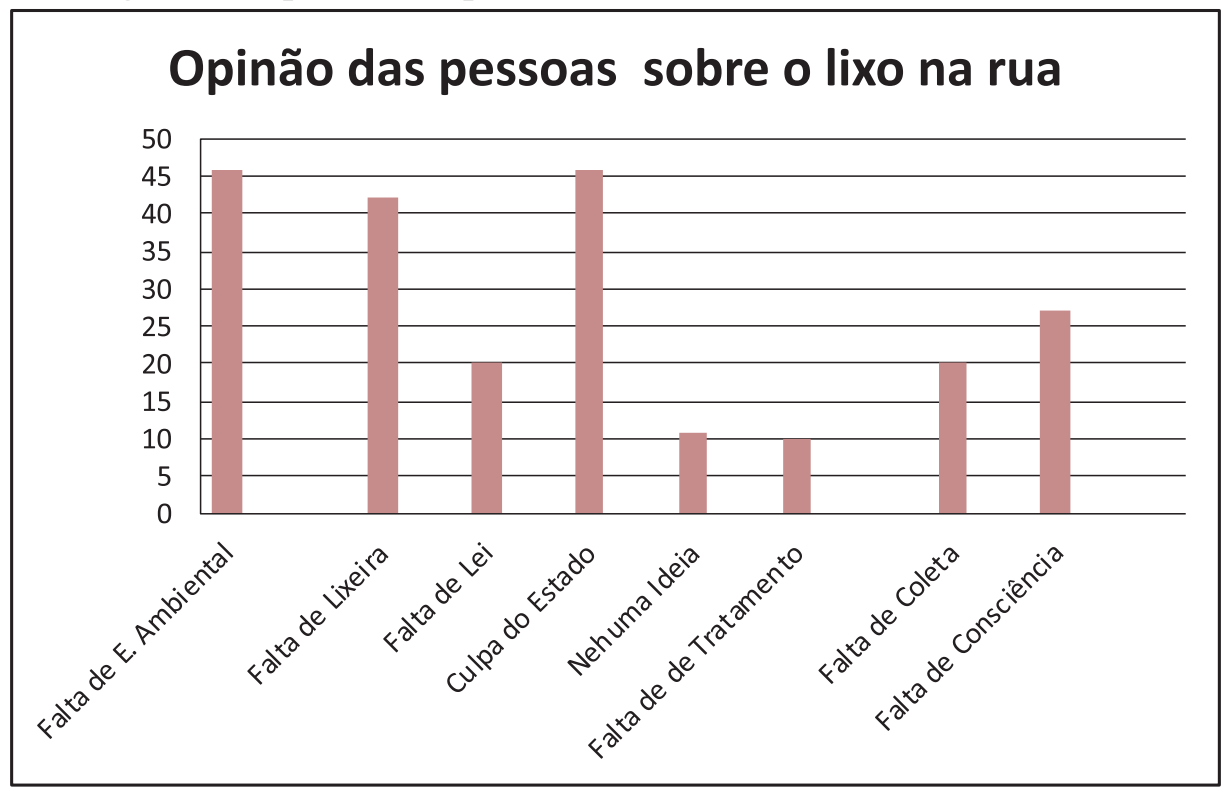

Fonte: Elaborado pelo autor através dos dados do campo

A última pergunta era o que deveria ser feito para melhorar essa situação? É uma pergunta aberta para verificar se existem correlações entre o perfil dos entrevistados e o impacto observado. Assim, 39 pessoas responderam que a melhor forma de lidar com os resíduos sólidos é colocar lixeiras nas ruas, dessa maneira não haverá necessidade de jogar lixo na rua, 23 acham que a única maneira de impedir que a população coloque lixo na rua é a aplicação de leis severas, ou seja, quem joga lixo na rua vai para a cadeia e deve pagar uma taxa muito alta. Para um aluno do ensino médio, a aplicação de leis severas é a única medida a ser aplicada para terminar essa prática, "porque ninguém quer ficar preso mesmo de for por uma hora".

A figura VII ilustra impressão das pessoas entrevistadas sobre o que deveria ser feito para terminar com o problema de lixo nas ruas em Cabaret. 
Ralph Charles, Regina Célia de Oliveira, Pedro Spanghero. The main consequences of solid waste on the environment and the health of the population in the municipality of Cabaret-Haiti

Figura 7. Sugestões das pessoas entrevistadas para melhorar a situação

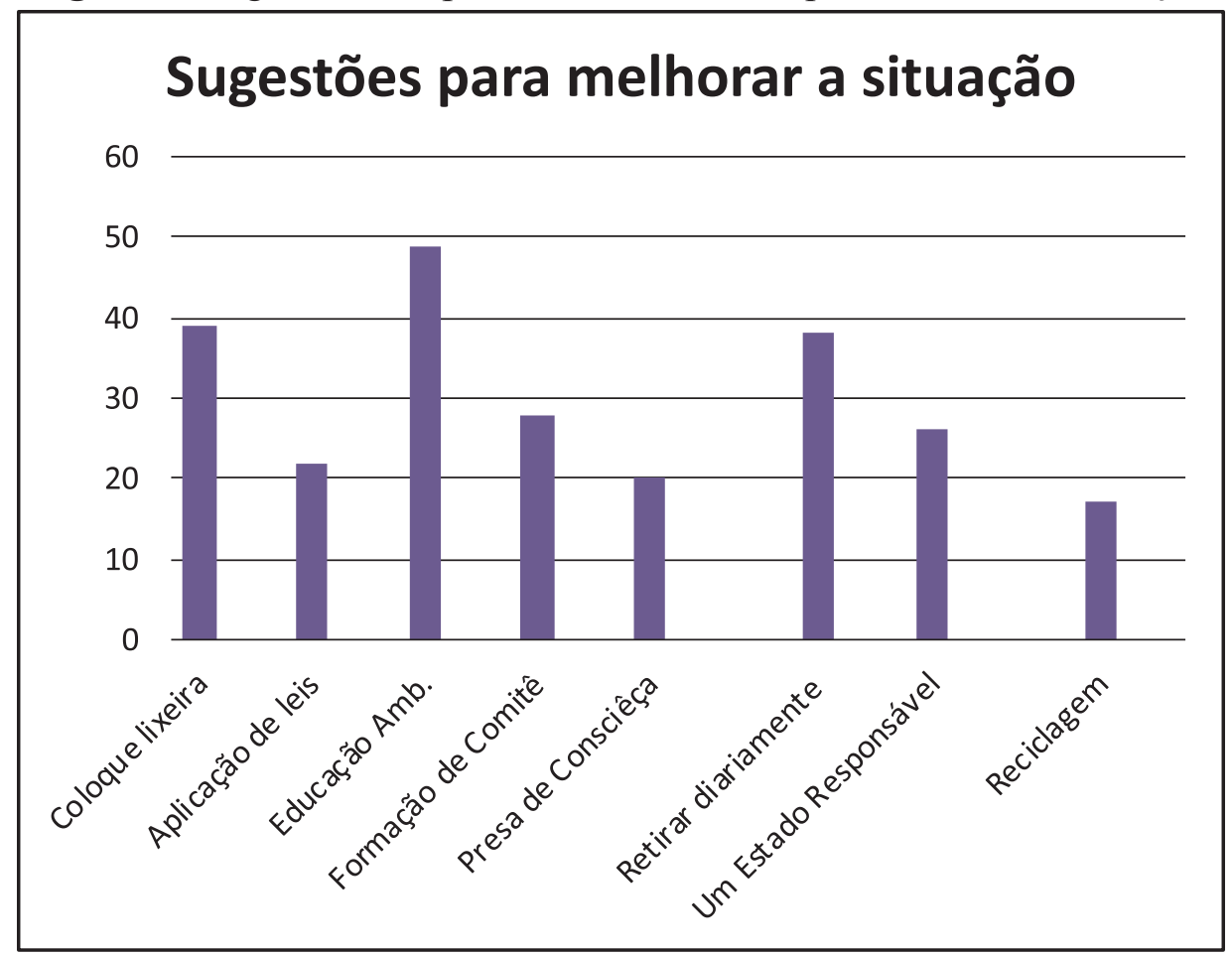

Fonte: Elaborado pelo autor através os dados do campo

As respostas obtidas durante a nossa entrevistas mostram de maneira clara algumas causas dos problemas ambientais, e baseando-se nos fatos que vivenciamos durante o trabalho de campo, concluímos que os resíduos sólidos são a causa de muitos problemas ambientais do município de Cabaret. É uma zona que apresenta fragilidade ambiental muito grande associada aos desastres naturais que ocorrem nos 10 últimos anos. . Não há presença de um sistema de saneamento básico, não existe nenhuma ação de tratamento de esgoto, isso faz com que a cada chuva, há probabilidades de inundações e quase todas as atividades socioeconômicas sejam paralisadas, especialmente as escolas. É um município que deveria ser considerado como uma área de risco, pois, está sujeito os riscos naturais e aqueles decorrentes das ações antrópicas. É importante que algumas medidas sejam adotadas para melhorar essa situação para evitar problemas mais graves no futuro sobre o meio ambiente e a saúde humana. 
Ralph Charles, Regina Célia de Oliveira, Pedro Spanghero. As principais consequências dos resíduos sólidos sobre o meio ambiente e a saúde da população no município de cabaret-haiti

\section{Considerações finais}

Conforme os dados e as discussões levantadas pela pesquisa, na prática, a quase totalidade dos municípios do território haitiano não possui um sistema adequado de tratamento de resíduos sólidos. A falta de políticas públicas desencadeia na deposição de lixo por parte da população em diversas zonas da cidade, pode-se afirmar que tal processo desponta como origem na perda da qualidade da saúde socioambiental. Uma solução para a problemática seria um fomento para criação de empresas de transformações de resíduos em matérias primas para usos diversos, considerando exemplos de diversas cidades no mundo. Os ganhos poderiam perpassar a composição da paisagem urbana, diminuindo o volume dos lixos a céu aberto e chegando até geração de emprego para a população. Por outro lado, teremos um ambiente mais sustentável, com menos poluição, diminuição na quantidade de emissão de dióxido de carbono, sendo que, durante a atividade de campo, a prática mais utilizada para eliminar os resíduos é a queimada a céu aberto sendo considera uma atividade inadequada, tendo em vista os diferentes impactos relacionados a essa prática.

Dentre as medidas que venham minimizar a situação observada, é sugerido um trabalho mais efetivo de educação e conscientização ambiental, bem como medidas eficazes que trazem resultados mais aparentes como reciclagem, compastagem, reflorestação, tratamento de esgoto, planejamento e controle do uso da terra, dentre outros. A adoção dessas ações pode garantir a médio e longo prazo um município de Cabaret em boas condições para o desenvolvimento de diversas formas de vida garantindo uma boa qualidade de vida para as futuras gerações.

\section{Referências}

AMORIM, R. R. ; OLIVEIRA, R. C. "Análise Geoambiental dos Setores de encosta da área urbana de São Vicente-SP”. Sociedade \& Nature$z a$, v. 19, p. 123-138, 2007.

AMORIM, R. R. ; OLIVEIRA, R. C. "Degradação ambiental e novas territorialidades no Extremo Sul da Bahia". Caminhos da Geografia (UFU. Online), v. 8, p. 18-37, 2007.

AMORIM, R. R ; PRADES, T. O. B. "Vulnerabilidade das Áreas Sujeitas as Inundações no Município de São João Da Barra, RJ, Brasil”. Revista de Departamento de Geografia, Universidade de São Paulo. São Paulo, Maio de 2017. 
Ralph Charles, Regina Célia de Oliveira, Pedro Spanghero. The main consequences of solid waste on the environment and the health of the population in the municipality of Cabaret-Haiti

ABNT - Associação Brasileira de Normas Técnicas. ABNT NBR 10004. "Resíduos Sólidos - Classificação", 2010.

ALMEIDA, C. M. "Compostagem" Programa de pós-graduação em solos e nutrição de planta. Universidade de São Paulo, Maio de 2008.

ALTAPRESSE " Cabaret ravagée par Le cyclone IKE, 8 septembre 2008. IBGE - Instituto Brasileiro de Geografia e Estatística. Pesquisa Nacional de Saneamento Básico. Rio de Janeiro: IBGE, 2010.

IHSI -- Institut Haitien de Statistique de d'Informatique. "Les comunes et Villes d'Haiti'. Port au Prince, fevrier, 2010.

Ministère de l'Education Nationale et de la Formation Professionelle (MENFP) "La formation continue des personnes administratifs, sociaux et de santé". Port-au-Prince, fevrier 2010.

RODRIGUES, J. M. ; SILVA, E.V. ; CAVALCANTI, A. P. B. (0rgs.) "Geoecologia das paisagens: uma visão geosistêmica da análise ambiental". Fortaleza: UFC, 2010.

ONU -- Organização das Nações Unidas. “O problema do lixo: Cidades do mundo produzem mais 10 bilhões de toneladas de resíduos todos os anos". Brasil, 7 de setembro de 2017.

ONU- Organisations des Nations Unies. "Cabaret dévasté apres le passage des cyclones Ike e Hanna”. Septembre, 2008.

ONU et la prévention des risques de catastrophe. Mars, 2010.

Plan d'Investissement de Cabaret "Plan d'investissent municipal: version proviso ire (PIMVP)" Republique d'Haiti, departement de l'oeust, comune de Cabaret, decembre 2001.

RODRIGUES, J. M. ; SILVA, E.V. ; CAVAlCANTI, A. P. B. (Orgs.) "Geoecologia das paisagens: uma visão geosistêmica da análise ambiental”. Fortaleza: UFC, 2010. 